\begin{abstract}
¿Cómo citar el artículo?
Sierra-Ospina, N., Lopera-Medina, S., Henao, C., y Quintero-Pulgarín, V. (septiembre-diciembre, 2020). Interpretación y apropiación de una política lingüistica: voces de estudiantes del área de la salud en una universidad pública. Revista Virtual Universidad Católica del Norte, (61), 42-65., https://www.doi.org/10.35575/rvucn.n61a4
\end{abstract}

\title{
| Interpretación y apropiación de una política lingüística: voces de estudiantes del área de la salud en una universidad pública
}

\author{
Interpretation and appropriation of a language policy: health science \\ students' voices in a public university
}

\section{Nelly Sierra Ospina}

Doctora en Educación

Escuela de Idiomas, Universidad de Antioquia

Medellín, Colombia

nelly.sierra@udea.edu.co

Orcid: https://orcid.org/0000-0002-8494-7394

CVLAC: https://scienti.minciencias.gov.co/cvlac/visuali-

zador/generarCurriculoCv.do?cod_rh $=0000188050$

\section{Sergio Lopera Medina}

Doctor en Lingüística

Escuela de Idiomas, Universidad de Antioquia

Medellín, Colombia

sergio.lopera@udea.edu.co

Orcid: https://orcid.org/0000-0003-2773-0890

CVLAC: https://scienti.minciencias.gov.co/cvlac/visuali-

zador/generarCurriculoCv.do?cod_rh=0000254665

\section{Catalina Henao}

Magíster en Letras: Lingüística

Facultad de Economía, Universidad de Antioquia

Medellin, Colombia

282cata@gmail.com

Orcid: https://orcid.org/0000-0003-1739-3587

CVLAC: https://scienti.minciencias.gov.co/cvlac/visuali-

zador/generarCurriculoCv.do?cod_rh $=0001804702$

\section{Víctor Quintero Pulgarín \\ Estudiante Licenciatura en Enseñanza de Lenguas Extranjeras \\ Escuela de Idiomas, Universidad de Antioquia Medellín, Colombia \\ victorm.quintero@udea.edu.co \\ Orcid: https://orcid.org/0000-0001-8091-9671 \\ CVLAC:https://scienti.minciencias.gov.co/cvlac/visuali- zador/generarCurriculoCv.do?cod_rh=0001804813}

Recibido: 04 de marzo de 2020

Evaluado: 04 de mayo de 2020

Aprobado: 11 de agosto de 2020

Tipo de artículo: Investigación científica y tecnológica.

\section{Resumen}

En este artículo se presentan los resultados de una investigación cualitativa sobre los procesos de interpretación y apropiación del estudiantado del programa de formación en lengua extranjera en una universidad pública de Medellín, Colombia. Esta es una etnografía de política lingüística, cuya información se obtuvo a través del análisis de documentos, un cuestionario, observaciones de clase y el portafolio. Los hallazgos evidenciaron que para unos/unas estudiantes el programa representa aprender inglés, y para otros/as es, entre otras concepciones, un material didáctico. Sus voces revelaron tanto aceptación como resistencia; por un lado, aceptación frente a la inclusión del inglés en el currículo, a la metodología, materiales del curso y al uso del portafolio como herramienta evaluativa; por otro lado, se encontró resistencia ante una de las estrategias de evaluación, ante la clase de inglés y la metodología de algunos/algunas docentes. Se concluye que estas voces presentan encuentros y desencuentros. La formación en inglés y la metodología propuesta, centrada en el estudiante, son bien valoradas por el estudiantado, pero aún prevalece una percepción de la lengua extranjera como una materia de poca importancia y aspectos motivacionales que el profesorado y la institución deben encarar con mayor vehemencia.

Palabras clave: Educación superior; Estudiante universitario; Lengua extranjera; Política lingüística. 


\section{| Abstract}

This article presents the results of a qualitative research on the processes of interpretation and appropriation of students on the institutional program of training in foreign language in a public university in Medellin, Colombia. This is an ethnography of language policy. The information was obtained through document analysis, a questionnaire, class observation and a portfolio. The findings show that for some students the program means to learn English, for others, didactic material, among other concepts. Their voices reveal acceptance, but also resistance. On the one hand, acceptance of the inclusion of English in the curriculum, the methodology, the course materials and the use of the portfolio as an evaluation tool. On the other hand, there is resistance to one of the assessment strategies, to the English class and the methodology of some teachers. It is concluded that these voices present encounters and disagreements. The English training and the proposed methodology, centered on the student, are well valued by the students, but it still prevails a perception of the foreign language as a subject of little importance and also motivational issues that teachers and the institution must face with greater vehemence.

Palabras clave: Higher education; University student; Foreign language; Language policy.

\section{| Introducción}

El mundo de la educación superior ha tenido cambios acelerados en los últimos tiempos, enfrentando las presiones de la globalización, la mercantilización de la educación y la crisis económica (Bretxa \& Vila, 2015; Villalobos et al., 2016). Debido a la globalización, el aprendizaje del inglés se ha convertido en una necesidad apremiante, no sólo en el ámbito educativo, sino también empresarial, y ha incursionado en contextos donde solía estar ausente. Algunos autores y autoras afirman que el inglés se habla en muchos países y es la lengua que más se aprende en el contexto internacional (Fishman, 2002; Kachru \& Nelson, 2001; Seidlhofer, 2011; Widdowson, 1997). Roldán y Peláez (2017) sostienen que, debido a su condición de idioma internacional, el inglés se convierte en la lengua primaria de aprendizaje de muchas instituciones educativas, y es por eso que dichas instituciones lo eligen como parte de su política lingüística en los planes de estudio.

Tal es el caso de una universidad pública en Medellín-Colombia, ya que en aras de ofrecer una formación más integral al estudiantado de pregrado de la institución y de responder mejor a las demandas del medio académico y laboral, la administración central se propuso repensar y establecer un programa de formación en lengua extranjera. La política no solo busca promover el aprendizaje de una lengua extranjera en los y las estudiantes de pregrado, sino también fomentar la internacionalización, impulsar el intercambio de saberes y culturas, y procurar mejores oportunidades profesionales para el estudiantado. Así mismo, define el inglés como lengua extranjera obligatoria para los y las estudiantes de pregrado de la institución, por su lugar fundamental en el ámbito académico y científico del mundo contemporáneo. El programa institucional cubre la formación de inglés para los programas que conducen a títulos de técnicos, tecnólogos y profesionales. 
Dicho programa se enmarca en la enseñanza del inglés con propósitos académicos generales (English for General Academic Purposes; EGAP por su sigla en inglés), en el cual se integran las diferentes habilidades de la lengua extranjera: habla, escucha, escritura y lectura; a través de la metodología Enseñanza de la Lengua Basada en Tareas (Task Based Language Teaching; TBLT por su sigla en inglés), centrada en tareas comunicativas, con temáticas y actividades del ámbito educativo y personal. Asimismo, se propone la integración con las tecnologías de la información y comunicación (TIC) y la articulación transversal del inglés a la oferta de materias de contenido disciplinar avanzado, en los diferentes programas académicos.

Con la intención de contribuir a la literatura existente sobre las políticas lingüísticas en el contexto de la educación superior, el presente estudio se centra en los procesos de interpretación y apropiación (Johnson, 2013a) de los agentes de la política. Dichos procesos son clave, ya que al visibilizarlos es posible comprender cómo se ejecuta realmente la política. Los agentes a analizar en este caso serán estudiantes de pregrado de una universidad pública en Medellín, Colombia, quienes se forman en una lengua extranjera en el programa de inglés de dicha universidad. Las preguntas de investigación que guían el presente trabajo son: ¿Cómo interpretan los y las estudiantes de pregrado el programa de formación de lengua extranjera?, ¿cómo los y las estudiantes de pregrado han apropiado el programa de formación en la lengua extranjera?

\section{| Marco teórico}

Este trabajo se ha nutrido de los elementos teóricos relacionados con la política lingüística y, en particular, de la política lingüística en el contexto de la educación.

\section{Política lingüística}

Esta investigación se ha apoyado en Johnson (2013a), quien desde una perspectiva sociocultural ha pensado la política lingüistica como un mecanismo que impacta la estructura, la función, el uso o la adquisición de una lengua. Además, dicho autor agrega que la política lingüística implica varios elementos en los que se encuentra una reglamentación oficial, la cual puede influenciar oportunidades de tipo económico, político y educativo. También, implica mecanismos implícitos o impuestos, los cuales determinan el uso de lengua y la interacción en contextos como la comunidad, el trabajo y la escuela. Así mismo, la noción de política no solo involucra un producto, sino también un proceso, el cual es generado por los agentes de la política en las diferentes esferas de creación, interpretación y apropiación. Y, por último, se deben tener en cuenta los textos de la política y discursos en diversos contextos, los cuales son influenciados por las ideologías y discursos de un contexto específico. Por tanto, la visión de Johnson, sobre política lingüística, permite considerar tanto sus elementos del nivel macro, tales como el análisis de la política, documentos, planes y programas, así como los elementos del nivel micro, entre los que se encuentran la puesta en marcha de la política y su relación con los diferentes agentes a nivel local (Liddicoat \& Baldauf, 2008).

Respecto a la puesta en marcha de las políticas lingüísticas, Johnson (2013a) llama la atención sobre los procesos de interpretación y apropiación de los agentes de la política lingüística, en aras de reconocer el poder que ejercen en el contexto local. De acuerdo con el autor, ambos procesos van de la mano, puesto que la manera como se interpreta la política determina cómo esta es apropiada por los agentes. En este orden de ideas, Levinson y Suton (2001, como se citó en Johnson, 2013a) sugieren el proceso de apropiación para enfatizar el lugar importante que los múltiples actores tienen en el proceso de la política. Así las cosas, la 
apropiación describe la manera como la política es puesta en acción, es decir, la forma como los agentes la definen y la aplican. Además, Johnson (2013a) sugiere que en la apropiación se pueden dar procesos de implementación, re-creación, desatención o resistencia por parte de los agentes. El autor agrega que se pueden presentar diferentes actividades de apropiación, las cuales incluyen: i) rechazo explícito: los participantes se rehúsan a obedecer una política lingüística en particular; ii) rechazo implícito: los participantes dan la apariencia de obedecer una política de lengua en particular; iii) adopción en términos de cambio de prácticas educacionales: se incluyen adaptaciones que involucran pruebas específicas, idiomas determinados, programas, currículos, entre otros; iv) adopción en términos de cambio de prácticas de negocios o servicios: se incluyen criterios de suficiencia para contratar o despedir docentes, incluir o excluir ciertos idiomas, inclusión de requisitos, entre otros; y v) adopción creativa: los participantes apropian una política lingüística de una forma creativa para continuar con una política existente.

Por tanto, se encuentra que en este proceso los agentes asumen una posición activa y tienen un lugar en la política, al hacer la lectura de la misma. Es decir, ya no son solamente implementadores/as de la política, sino que son también creadores (Menken \& García, 2010), dependiendo de la interpretación que hayan realizado de esta.

\section{Política lingüística en educación}

Liddicoat (2013) señala que las políticas lingüísticas en educación juegan un papel importante en las maneras como una sociedad articula y planea el futuro de sus miembros. Igualmente, indica que estas políticas pueden encontrarse, de manera explícita, articuladas en documentos oficiales, pero también pueden darse de manera encubierta en los supuestos y prácticas, en relación no solo con el uso de una lengua, sino también en el aprendizaje de una lengua en el contexto educativo. Además, Liddicoat plantea que las políticas del Gobierno en educación frecuentemente hacen referencia a los asuntos relacionados con la lengua.

Las políticas lingüísticas en educación son un mecanismo utilizado para crear, en las instituciones educativas, prácticas lingüísticas de manera fáctica (de facto o "de hecho"). Es de esta forma como, en escuelas y universidades, se llevan a cabo las decisiones respecto a políticas lingüísticas relacionadas con la lengua materna, una lengua extranjera o una segunda lengua (Shohamy, 2006). En este orden de ideas, Liddicoat (2013) presenta cuatro tipos de políticas lingüísticas en educación: las políticas educativas sobre la lengua oficial, las políticas educativas en relación con la enseñanza de una lengua extranjera, aquellas relacionadas con las lenguas de las minorías y las que tienen que ver con la expansión de lenguas externas. El presente estudio se enfocará en las implicaciones de las políticas educativas, en relación con la lengua extranjera inglés en los programas que conducen a títulos profesionales. Al respecto, Liddicoat (2013) plantea que la mayoría de las sociedades tienen políticas sobre la enseñanza y aprendizaje de una lengua adicional en el sistema educativo. Estas políticas implican el aprendizaje de lenguas que no se hablan en la sociedad y que su adquisición se da a través del sistema educativo.

Por su parte, Johnson (2013a) señala que las políticas lingüísticas en educación describen las políticas oficiales y no oficiales creadas a lo largo de las múltiples capas de las políticas y contextos institucionales, los cuales van desde las organizaciones nacionales hasta el aula de clase, e impactan el uso de una lengua en el aula y en la escuela. Igualmente, agrega que las políticas lingüísticas en educación son interpretadas y apropiadas de maneras creativas e impredecibles en el aula de clase, en la escuela y en la comunidad. Dado el reconocimiento a la agencia de los diferentes agentes de las políticas lingüísticas en educación, Johnson (2013a) llama en sentido figurado árbitros de la política a todos los individuos que puedan tener 
influencia sobre estas; es decir, un árbitro de la política ejerce poder en la manera como esta es creada, interpretada, apropiada. Es así como en este estudio se toma el concepto de "árbitros" del autor para referirse al estudiantado (D. Johnson, comunicación personal, 31 julio de 2018) de la universidad, dado que pueden considerarse como tal en la implementación dentro del aula de clase.

\section{Estudios previos}

La literatura proveniente de los diferentes contextos, nacional e internacional, refleja el interés y la preocupación por el análisis y estudio de las políticas lingüísticas, enfocadas a la enseñanza y aprendizaje del inglés. En el ámbito internacional se encuentran algunos estudios, en relación con las percepciones del profesorado sobre la política lingüística en diferentes niveles de la educación (Canale, 2015; Díaz et al., 2015; Fallon \& Rublik, 2012; Johnson, 2013b; Jung \& Norton, 2002). En el contexto de la educación superior, Alagozlu (2012) realizó un estudio en el cual analizó las percepciones de docentes de inglés sobre la política de instrucción del inglés como lengua extranjera en Turquía; en la misma línea, Wang (2008) exploró las percepciones de docentes de educación superior sobre la implementación de la política lingüística y el currículo en China. De igual manera, Kagwesage (2012) llevó a cabo una investigación sobre las reflexiones de los estudiantes universitarios sobre el uso del inglés como medio de instrucción en la institución, y Tseligka (2016) realizó un análisis de la política lingüística en Grecia sobre los factores que pueden posibilitar el éxito en su desarrollo e implementación. En la misma línea, Pereira (2013) presenta un estudio en el cual exploró las percepciones de administradores, docentes y estudiantes de pregrado sobre una política lingüística en una institución de educación superior. Por su parte, Tsuchiya \& Pérez (2015) investigaron las percepciones de estudiantes en España y Japón sobre la implementación del aprendizaje integrado de contenidos y lengua extranjera (AICLE; CLIL por su sigla en inglés) en la educación superior. También, Muñoz (2019) llevó a cabo una investigación sobre las creencias y actitudes lingüísticas del estudiantado universitario de las áreas de medicina, ingeniería civil y derecho. En cuanto a los procesos de apropiación de los estudiantes universitarios, se encuentra a Fuentes (2019), quien investigó sobre la manera como tres estudiantes bilingües/multilingües se han apropiado de la política lingüística en una universidad en los Estados Unidos.

De otro lado, en el contexto colombiano se encuentran algunas publicaciones sobre la implementación de la política lingüística nacional y percepciones de agentes de la política en la educación básica media (Bermúdez et al., 2014; Tejada y Samacá, 2012). En cuanto a los procesos de apropiación de las políticas lingüísticas, se podrían mencionar los estudios de Miranda (2018) y Peláez \& Usma (2017), los cuales se han llevado a cabo en el contexto de la educación básica secundaria, buscando explorar los procesos de apropiación de docentes y estudiantes en el aula, tanto en el contexto urbano como en el contexto rural del departamento de Antioquia. En el ámbito de la educación superior, Gómez (2017) realizó un estudio sobre las creencias de estudiantes universitarios, en relación con el aprendizaje del inglés; asimismo, Jiménez (2018) indagó sobre las percepciones de un grupo de estudiantes de inglés como lengua extranjera en una universidad pública, en relación con el aprendizaje del inglés y el nivel de compromiso durante el proceso. Igualmente, Usma et al. (2018) presentaron una investigación desde las voces de los/las estudiantes indígenas en la Universidad de Antioquia, en la cual se manifiesta la necesidad de pensar en principios pedagógicos más equitativos, a la par con una implementación y apropiación más sensible y efectiva de este tipo de reformas educativas en la educación superior. En la misma línea, Arismendi et al. (2016) investigaron acerca de las representaciones que tiene un grupo de estudiantes indígenas de una 
universidad pública, en relación con las lenguas materna y extranjeras. Adicionalmente, Alonso et al. (2015) presentaron un estudio sobre el impacto de la educación superior en el dominio del inglés en instituciones privadas y públicas. Finamente, Montoya (2013) planteó que las políticas y la planeación lingüística inciden en las actitudes lingüisticas de los alumnos colombianos.

A modo de conclusión, se podría decir que estas publicaciones dan cuenta del interés y preocupación de la comunidad académica del país sobre las políticas lingüísticas implementadas en el contexto colombiano de la educación superior. Sin embargo, es importante anotar que en el contexto colombiano poco se han visibilizado las voces de los agentes de las políticas lingüísticas, como las del estudiantado de educación superior, respecto a los procesos de interpretación y apropiación de las mismas. Como afirman Liddicoat \& Baldauf (2008) son pocos los estudios sobre los elementos de los niveles micro de las políticas lingüísticas, es decir, en relación con la implementación de las políticas y su conexión con los agentes locales, como el profesorado, el estudiantado y demás agentes del sistema educativo, en aras de comprender mejor los procesos en el ámbito local.

\section{| Metodología}

Este estudio se basó en los principios de la investigación cualitativa (Denzin \& Lincoln, 2008; Denzin \& Lincoln, 2018) para llevar a cabo una etnografía de política lingüística en diversos escenarios (Canagarajah, 2006; Hornberger \& Johnson, 2007; Hornberger \& Johnson, 2011; Johnson, 2013a; McCarty, 2011; McCarty, 2015). La etnografía de la política lingüística da cuenta de la manera como los agentes se relacionan o se involucran en los procesos de las políticas lingüisticas en las diferentes etapas que las conforman, esto es, en los procesos de creación, interpretación y apropiación. Aparte de lo anteriormente señalado, Johnson \& Johnson (2014) plantean que la etnografía de una política lingüística posibilita la manera como la política macro se relaciona con las prácticas educativas; de igual manera, Hornberger y Johnson (2007, 2011) indican que la investigación etnográfica de la política lingüística es un medio para explorar la manera cómo las diversas interpretaciones locales, implementaciones y negociaciones, y posiblemente resistencias, pueden conllevar a promover espacios abiertos para la educación multilingüe. Por tanto, en la etnografía de la política lingüística se encuentra una posible ruta alternativa para analizar el proceso de interpretación y apropiación del estudiantado de la institución, desde la perspectiva émica, es decir, desde la perspectiva de los y las participantes del estudio. Además, permitirá dar cuenta del proceso de implementación de una política lingüistica a la cual una universidad pública le ha apostado para ofrecer una formación más integral a todo el estudiantado.

\section{Contexto y participantes}

El estudio se llevó a cabo en las unidades académicas de las diferentes áreas del conocimiento, ciencias exactas y naturales, ciencias sociales y humanas, y área de la salud, de una universidad pública en Medellín, Colombia. Los y las participantes de este estudio fueron estudiantes de pregrado, de programas conducentes a título profesional de la institución. El estudiantado lo conformaron tres grupos por cada área de conocimiento, quienes se encontraban tomando cursos de inglés en el programa de formación en lengua extranjera. Este artículo presenta los hallazgos de la información obtenida del estudiantado del área de la salud, específicamente estudiantes de pregrado que se encontraban en los cursos de inglés, nivel 1 y 3 , de las Facultades de Medicina, Enfermería y Veterinaria. 


\section{Métodos de recolección de la información}

La recolección de información se hizo a través del análisis de documentos (Heck, 2004; Merriam, 1998), la observación directa no participativa (Angrosino, 2015), un cuestionario (Dornyei \& Taguchi, 2010; Johnson \& Christensen, 2008) y el portafolio (Merriam, 1998). La recolección y análisis de la información siguió un proceso específico. En primer lugar, se llevó a cabo el análisis de documentos oficiales que incluyeron el documento maestro del programa de formación de lengua extranjera y los programas de curso de los dos niveles de inglés. El paso siguiente fue la observación de clases de los profesores que aceptaron participar en la investigación. Se observó aproximadamente el 50 \% de las clases (32 horas) de cada uno de los tres cursos de inglés en tres facultades del área de la salud durante el semestre 2019-1. Estas observaciones tuvieron como objetivo examinar los procesos de apropiación que se dan dentro del salón de clase por parte de las y los estudiantes universitarios. Al finalizar las observaciones, se aplicó un cuestionario con preguntas abiertas y cerradas en el que se les preguntó a las y los estudiantes por su interpretación, sus percepciones y sentimientos relacionados con la política lingüística (ver Apéndice A). El cuestionario fue respondido por un total de 39 estudiantes del área de la salud, que estaban matriculados en los cursos de inglés durante el semestre 2019-1. Este grupo lo conforman 11 estudiantes de inglés I, de la Facultad de Medicina; 13 estudiantes de inglés I, de la Facultad de Veterinaria; y 15 estudiantes de inglés III, de la Facultad de Enfermería. Posteriormente, en aras de revisar los elementos que evidenciaran el proceso de apropiación de la política en los y las estudiantes, se recolectaron los portafolios de 15 participantes clave, quienes, además, respondieron dos preguntas respecto a sus experiencias con esta estrategia evaluativa.

Los y las participantes clave fueron aquellos estudiantes cuya participación, proactiva o distante, fue llamativa durante las observaciones de clase. Finalmente, para el análisis de la información se aplicó el proceso inductivo para la codificación y la generación de las posibles categorías o temas, con el objetivo de buscar respuestas a las interpretaciones y a la manera cómo el estudiantado ha interpretado y apropiado la política lingüística implementada en la universidad.

\section{Hallazgos}

A continuación, se presentan las voces del estudiantado, las cuales dan cuenta de la manera como ellos y ellas han interpretado y apropiado la política lingüística de la institución.

\section{Interpretación del programa de formación en lengua extranjera}

En el cuestionario (ver Apéndice A), al preguntar al estudiantado sobre lo que para ellos/ellas significa el programa institucional, se encontró que, en general, lo perciben como un programa innovador y dinámico para el aprendizaje de otras lenguas y para el desarrollo de las diferentes habilidades de la lengua. Para una estudiante en Medicina, por ejemplo, el programa es "Actividades didácticas y creativas diseñadas para un aprendizaje ameno de una segunda lengua"; en el caso de Veterinaria, un estudiante responde "Suena a que es un programa sofisticado"; y en Enfermería, una estudiante dice: 
Han sido unas experiencias muy enriquecedoras porque me permiten conocer mucho más y a profundidad todo el idioma inglés. Han sido procesos muy dinámicos y vivenciales que nos llevan a apropiarnos de ese idioma, las clases no se quedan únicamente en la teoría.

Así mismo, para otros y otras estudiantes el programa de inglés corresponde a recursos didácticos del programa; para un estudiante de Enfermería, el programa es "Las cartillas con las que se guía el programa"; de igual manera, en Medicina otro estudiante dice que es "Una cartilla de inglés"; y en el caso de Veterinaria, una estudiante responde "Es un folleto para aprender inglés". En otras palabras, mientras que para algunos/algunas estudiantes el programa significa aprender una lengua extranjera, para otros este se limita a ser un material didáctico.

Aparte de conocer lo que le significa el programa al estudiantado, en el cuestionario se preguntaba también sobre su experiencia estudiando la lengua extranjera en el programa. En sus voces se encontró que para una estudiante de la Facultad de Veterinaria la experiencia es "Buena, la clase es dinámica y para nada tediosa"; también, en Medicina una estudiante considera que "La experiencia ha sido buena. A pesar de ser el primer nivel que curso, el aprendizaje y la experiencia en general han sido muy buenos"; y en el caso de Enfermería, "Podría considerarse como buena pues implementa objetivos realistas y acordes al nivel en el que se debe ubicar un estudiante universitario a través de los diferentes niveles". En sus voces se percibe que, al describir su experiencia en el programa, se remiten directamente a lo que han vivido en el aula de clase y a los objetivos propuestos en el programa; además, se evidencia que su experiencia ha sido grata.

Uno de los argumentos de la política lingüistica de la universidad es ofrecer una formación más integral al estudiantado de pregrado de la institución y de responder mejor a las demandas del medio académico y laboral. En las respuestas del cuestionario, el estudiantado se alinea a este argumento y reportan que para ellos y ellas aprender inglés representa: "Mayor habilidad para desempeñarse en ámbitos académicos y laborales" (Estudiante Veterinaria), "Significa una gran herramienta para desempeñarnos mejor en otros ámbitos laborales, académicos y desempeño en otros países" (Estudiante Enfermería), y finalmente "Tener acceso a información disponible que está en inglés, lo que permite ampliar el conocimiento, ampliar la gama de oportunidad laboral, viajar al extranjero para intercambios, independencia y seguridad" (Estudiante Medicina). Al respecto, se señalan dos puntos: uno, es evidente el lugar de reconocimiento del inglés en la comunidad estudiantil; el otro es que esta lengua mantiene un predominio frente a las otras lenguas extranjeras existentes, puesto que en el medio se encuentra en ella un camino efectivo para formarse de manera integral y con ello asegurarse un mayor éxito laboral y académico. Lo anterior, ha sido fuertemente promovido, tanto en la política lingüística nacional (Ministerio de Educación Nacional -MEN-, 2005) como en la misma política institucional de la universidad pública.

Del mismo modo, algunos de los principios de la política involucran mejorar el nivel de desempeño del estudiantado, articular el inglés de manera transversal en los cursos disciplinares y que sean independientes y autónomos con el aprendizaje. Al respecto, el 90 \% de este grupo de estudiantes está de acuerdo con estos principios y ve al programa institucional como una gran oportunidad académica y de formación en la autonomía. Lo anterior, lo corroboran sus voces al señalar los beneficios que tiene el portafolio, una de las estrategias de evaluación, dado que contribuye al aprendizaje autónomo y a la reflexión:

Me parece que promueve bastante la autonomía y la reflexión debido a que alli es donde el estudiante se da cuenta de los conocimientos que tiene, si de verdad ha aprendido durante el proceso o si tiene falencias que debe de mejorar. (Estudiante Medicina) 
De igual manera, otro estudiante señala que "[El portafolio fortalece la autonomía] bastante; desde que se haga con responsabilidad se logran adquirir muchos conocimientos adicionales a los que se dan en clase" (Estudiante Enfermería). Y una estudiante responde sobre el portafolio "Sí lo promueve porque gracias a él se realizan las clases y se dejan las tareas para la casa para promover más en lenguaje" (Estudiante Veterinaria). En el documento maestro se encuentra establecido que el programa busca promover una evaluación alternativa, por lo cual sugiere el portafolio como uno de los instrumentos de evaluación. De acuerdo con las respuestas de los y las estudiantes se encontró que esta herramienta no tradicional de evaluación ha sido acogida de manera positiva y que la ven como una buena alternativa para aprender de manera independiente.

En líneas anteriores se indicó que el programa institucional sigue el enfoque metodológico TBLT; así que se le indaga al estudiantado por la manera cómo comprenden la metodología del programa, y algunos/algunas estudiantes la describieron así: "Un laboratorio de inmersión en el idioma extranjero mediante situaciones reales y dinámicas" (Estudiante Enfermería); "[Una metodología] bastante práctica, es poco el trabajo que se desempeña de forma magistral, es un proceso quizás más de complemento profesor-alumno, en el cual se evidencia la participación de ambos" (Estudiante Medicina); y otro comenta "Es bastante completa, ya que abarca temáticas reales a lo que tiene que ver con el aprendizaje del inglés y conversaciones" (Estudiante Veterinaria). Las palabras del estudiantado de estas tres Facultades muestran que la metodología les resulta innovadora, práctica y conectada con sus realidades.

En la misma línea, en el programa de inglés se encuentra planteado que las tareas fomentan la interacción alrededor de temas relacionados con la vida personal y educativa. Además, en el cuestionario se preguntó al estudiantado acerca de su percepción sobre las tareas, y estas son algunas de sus respuestas: "muy buenas, didácticas y de mucho aprendizaje" (Estudiante Veterinaria); "Interesantes y manejables" (Estudiante Enfermería); y "Muy apropiadas para tener un buen aprendizaje" (Estudiante Medicina). Se observa que las califican positivamente porque promueven el aprendizaje de una manera didáctica.

Teniendo en cuenta su comprensión del programa, también se indagó por la manera en que el estudiantado comprende su rol en las clases. Para una estudiante su papel en el programa es "Estar muy atento/a a aprender, preguntar siempre que no entienda, hacer mucho trabajo independiente como repasos, actividades, etc." (Estudiante Enfermería). Para otra estudiante, corresponde a "Participar activamente de las actividades que se estén realizando, mostrar interés en la clase y resolver dudas con mi profesor/a y de forma autónoma" (Estudiante Medicina). Y también "Recibir la información que me brindan y estudiar por fuera de la clase complementando mi aprendizaje" (Estudiante Veterinaria). De acuerdo con las respuestas del estudiantado sobre su rol en los cursos de inglés, se podría decir que hay dos elementos a destacar; el primero tiene que ver con su papel activo en el aprendizaje de la lengua en el aula de clase, y el segundo con la autonomía. Lo anterior va en consonancia con lo establecido en el documento maestro del programa, en el que se plantea que el estudiantado debe tener un rol más protagónico en su aprendizaje, lo cual se evidencia en su autonomía y en el control de su propio proceso de aprendizaje.

\section{Apropiación de la política lingüística}

Con base en Johnson (2013a), quien sugiere que en la apropiación se pueden dar procesos no solo de implementación, re-creación, desatención o resistencia, sino también actividades que incluyen rechazo y adopción, en este estudio se encontró que las voces del estudiantado reflejan procesos de aceptación, resistencia y adaptación en la puesta en marcha de la política lingüística de la institución. 
Aceptación. Teniendo en cuenta que la institución determinó el inglés como la lengua extranjera de carácter obligatorio para los programas de pregrado, se les preguntó a los y las estudiantes sobre su nivel de aceptación frente a esta decisión institucional y se encontró que en general el estudiantado del área de la salud está de acuerdo con dicha decisión, ya que, de 39 encuestados, 20 respondieron que están "de acuerdo" y 19 "totalmente de acuerdo." En relación con la inclusión de los cinco niveles de inglés en los planes de estudio, se halló que la mayoría del estudiantado de las tres facultades también acepta este cambio en los programas de formación. Al respecto, el 84.7 \% muestra su acuerdo.

Igualmente, en las observaciones de clase fue evidente que el estudiantado acepta las actividades propuestas por el/la docente del curso. En Veterinaria, por ejemplo, el/la docente le solicitó al grupo de estudiantes traer copia del material para la unidad 1, les asignó completar un cuadro sobre la rutina diaria y, quienes estaban en ese momento en el aula, procedieron a realizar el ejercicio con una actitud receptiva. De igual manera, en el curso en Enfermería el/la docente solía iniciar siempre la clase preguntando al grupo por las actividades que habían realizado en días anteriores y, dado que esta era una actividad habitual en el curso, el estudiantado siempre respondía de manera proactiva al disponerse a preparar su respuesta. Lo mismo se evidenció en el curso de la Facultad de Medicina, cuando el/la docente indicaba al grupo las actividades a incluir en el portafolio, el estudiantado tomaba nota y corroboraba con el/la docente. Además de lo anterior, se preguntó al estudiantado por los recursos que utilizaba para el aprendizaje del inglés. De acuerdo a sus respuestas, se observa que los materiales más utilizados son los propuestos en el programa, tales como videos, audios y el material de apoyo diseñado, lo cual igualmente refleja la aceptación de la política.

Otra fuente de información que apoya que el estudiantado reconoce la política lingüística son las observaciones de clase. Los investigadores y las investigadoras notaron que la mayoría de los y las estudiantes utilizan fotocopias, documentos en formato pdf, celulares, computadores portátiles y tabletas, en las que trabajan con el material de apoyo del programa institucional dentro del aula. Además, el estudiantado, por lo general, trabajaba activamente en las actividades propuestas por el/la docente en el aula de clase.

Por otro lado, y en relación con las actividades desarrolladas en el curso, en el cuestionario el estudiantado expresó su conformidad y aprendizaje. Al respecto, un estudiante en Medicina expresa "Me sentí muy a gusto en cada actividad, me divertí y aprendí situaciones y conceptos nuevos que a lo largo de mi vida no había aprendido"; en la misma línea, en Enfermería un estudiante comenta "I think that I have improved my speak and vocabulary, I pay attention in class and with the class strategy about the last weekend I could practice the past tense" [Yo pienso que yo he mejorado el habla y el vocabulario, yo pongo atención en clase y con la última estrategia acerca del fin de semana yo pude practicar el pasado simple]. Y en Veterinaria "Mi experiencia con el portafolio fue considerablemente buena, aprendí con las actividades aplicativas y reforcé conocimientos que ya tenía. Aunque, en ciertas ocasiones, encontraba complicadas las actividades para alguien que no ha tenido ningún acercamiento al inglés". En ese sentido, es evidente tanto el sentimiento de agrado en el programa como también su valoración frente a los aprendizajes ganados en el proceso.

También, en el cuestionario, manifestaron que llevar el portafolio ha sido una experiencia positiva y de aprendizaje. Al respecto, reportan: "Mi experiencia con el portafolio fue considerablemente buena, aprendí con las actividades aplicativas y reforcé conocimientos que ya tenía. Aunque, en ciertas ocasiones, encontraba complicadas las actividades para alguien que no ha tenido ningún acercamiento al inglés" (Estudiante Veterinaria). Así mismo, este testimonio narra "Ha sido una experiencia muy fructífera, ya que la metodología empleada con el laboratorio además de fortalecer lo visto en clase permite trascender de este aprendizaje y adentrarse de maneras diferentes en temas relacionados" (Estudiante Enfermería). Y otra estudiante responde: 
Me parece que es un buen material de apoyo debido a que se puede colocar en práctica todos los temas aprendidos, además de que se le realiza un seguimiento para saber cómo se ha avanzado y qué dificultad tiene el estudiante con cada tema tratado en clase. (Estudiante Medicina)

A modo de conclusión, las voces del estudiantado reflejan su reconocimiento y valoración al portafolio que llevan en los cursos de inglés.

Resistencia. En contraste, se percibió que, si bien el estudiantado ha manifestado su aceptación frente a diferentes elementos del programa, también sus voces y sus actitudes en clase dan cuenta de su desacuerdo o resistencia frente a algunos elementos de los cursos del programa. En relación con la evaluación del programa, se evidenció un cuestionamiento a uno de los instrumentos de evaluación. Al preguntar por el Taller EGAP, el cual es una de las estrategias evaluativas, ellos y ellas respondieron: "Es algo que no aporta pues para su diseño no se tienen en cuenta las temáticas que se vienen trabajando, siempre son muy confusos pues dan por cierto que tenemos unos conocimientos previos específicos para esta prueba" (Estudiante, Enfermería). Otro estudiante señala:

Es muy diferente a lo que es el programa ya que tiene muchas más cosas y no permite que sea enseñado de formas más didáctica por lo que tanta información genera un poco de confusión y estrés en el estudiante. (Estudiante, Medicina)

En el mismo sentido, una estudiante dice: “Debe tener más tiempo para resolver ya que el párrafo era largo y duro de traducir, difícil, no acorde con el nivel" (Estudiante, Veterinaria). Adicionalmente, en la observación de clase en Medicina una estudiante dijo sobre el taller EGAP "es un método evaluativo obsoleto". De acuerdo al estudiantado se puede plantear que esta estrategia evaluativa se conecta poco con la propuesta metodológica del programa por la diferencia y complejidad en los temas propuestos, lo cual les ha generado un sentimiento negativo.

Por otro lado, la tensión con el taller se corroboró en las observaciones de clase. En Medicina, mientras el/la docente explicaba unos temas de gramática como preparación para presentar el taller EGAP, varios/varias estudiantes hacían otras actividades: uno miraba el celular, otro escribía en el WhatsApp, otra estudiante escuchaba algo en el teléfono celular, una estudiante se durmió en el puesto y otra estudiante se pasaba las manos por la cara como tratando de despertar. También, en la Facultad de Enfermería se observaron algunas reacciones del estudiantado que podrían reflejar su nivel de resistencia. En una clase, durante la explicación de unos temas de preparación del taller EGAP, un estudiante comenta con un tono como de incomodidad "Es difícil entender esta teoría". Al mismo tiempo, se observó que muy pocos estudiantes tomaban nota de la explicación, en su mayoría miraban al tablero en silencio.

Aparte de lo anterior, durante las observaciones de clase se notaron algunas actitudes de los y las estudiantes que podrían evidenciar resistencia con el curso de inglés. En la Facultad de Medicina, con frecuencia, se observaron actitudes de desánimo o desmotivación en el grupo. Incluso algunos de sus comentarios en el aula así lo evidenciaban; en una clase el/la docente dio una instrucción para realizar una actividad y un estudiante preguntó con un tono fuerte “qué hay que hacer?" y su compañero le respondió "ni idea", igualmente con una actitud de insatisfacción. Del mismo modo, en los cursos de Enfermería y Veterinaria, algunos/algunas estudiantes usaban recurrentemente el celular para propósitos diferentes a los académicos. También, se veía al estudiantado sentado en una posición relajada y con una actitud distraída, mirando fijamente al exterior del aula o a un punto fijo. Además, en repetidas ocasiones, un alto porcentaje del grupo llegó tarde a la clase y algunos/algunas estudiantes salían de clase antes de terminarse. Particularmente, en el curso de la Facultad de Veterinaria fue bastante evidente el alto porcentaje de inasistencia a clase. En este grupo estaban matriculados 20 
estudiantes, de los cuales solamente asistían a clase entre un $10 \%$ y $12 \%$. Únicamente el día del examen final estuvo el grupo completo. Respecto a esta situación, el último día de clase el/la docente llama la atención al grupo por su inasistencia a la clase:

Muchos de ustedes dicen 'yo no vine a estudiar inglés', cuando salgan muchos de ustedes no venían a clase, la vida se los va a cobrar ese engaño porque sacan 4.5 y no saben inglés, después les va a tocar pagar un curso o pagar una traducción por no aprovechar lo que la universidad les da gratis. (Observación de clase).

Teniendo en cuenta que la institución ha establecido el inglés como la lengua extranjera a incluir en los planes de estudio, llama la atención el comportamiento del estudiantado en el aula de clase en las diferentes facultades, especialmente en la Facultad de Veterinaria. Si bien en la pregunta 6 del cuestionario, 20 estudiantes (51\%) manifestaron estar de acuerdo con la elección del inglés, y 19 estudiantes (49\%) indicaron estar totalmente de acuerdo, su grado de compromiso con el aprendizaje de la lengua extranjera en estos cursos es poco coherente con sus respuestas a dicha pregunta.

Por otro lado, en las observaciones de uno de los cursos del área de la salud se encontraron algunas reacciones o comentarios del estudiantado que evidencia algún grado de resistencia con la metodología del profesorado. En un curso, algunos/algunas estudiantes manifestaron su desacuerdo frente a la posición del/de la docente en relación con la corrección de la pronunciación. En una de las clases, un estudiante le pide al/a la docente que le corrija la pronunciación, a lo cual el/la docente responde "La corrección inmediata es tradicional; en lo moderno se deja que el estudiante fluya". Ante esta respuesta el estudiante hace un gesto de negación y uno de sus compañeros añade "yo estoy de acuerdo en que se la corrija". De igual manera, en el cuestionario se le solicitó al estudiantado describir la metodología del programa, y se halló que algunas de sus respuestas evidencian algún grado de resistencia frente a la metodología utilizada en los cursos. Al respecto, hay un estudiante enfatizando el tema de la corrección "No estoy de acuerdo con que no se me corrija la pronunciación cuando me equivoco". Por otro lado, en otra facultad, un estudiante, al hablar de la metodología, la describe como "monótona y repetitiva en la entrega de los libros de trabajo", refiriéndose al material impreso del programa. En otra facultad se observó que una estudiante considera que la metodología es "aburrida dependiendo del profesor, hay docentes que hacen de esta experiencia algo enriquecedor, sin embargo, otros se ciñen a rellenar una cartilla". En este sentido, se evidencia que sus palabras remiten a la manera como el profesorado lleva a cabo la clase, y manifiestan su desacuerdo frente a la corrección de errores en clase y al uso constante de los materiales impresos del programa en la misma, los cuales, parece, se han convertido en el único recurso de clase y, por tanto, es la representación que el estudiantado tiene de la metodología del programa.

Adaptación. En las observaciones de clase el grupo de la investigación notó que el estudiantado con frecuencia propone realizar algunas actividades diferentes a las propuestas por el/la docente del curso. Tal es el caso del grupo de estudiantes de la Facultad de Veterinaria, quienes sugirieron al/a la docente trabajar con canciones en inglés al inicio de cada clase y él/ella accedió. Para ello, el/la docente iniciaba cada clase con un ejercicio de escucha a través de una canción en inglés, cada estudiante recibía la transcripción de la canción y debía enumerar las líneas del texto, según el orden de la misma. Dado el acuerdo con el grupo, el/la docente preguntaba “¿Alguien quiere una canción específica para la próxima clase?", a lo que, por ejemplo, una estudiante respondió "Take on me, profe". Por su parte en la Facultad de Medicina, un estudiante sugirió hacer talleres más dinámicos utilizando la plataforma Kahoot para jugar y aprender los contenidos del material del curso. En este caso, el estudiante realizó el taller sobre vocabulario de la familia y de la casa, y en clase lideró el juego explicándole a sus compañeros y compañeras la dinámica correspondiente. 
Por otro lado, un estudiante de Enfermería sugirió que se incorporaran otros tipos de materiales y no ceñirse tanto al material que propone el programa. Otro elemento que propone el estudiantado está relacionado con la gramática. Tanto en las observaciones de clase como en las encuestas, los estudiantes propusieron agregar o profundizar elementos gramaticales y no tomarlos tan superficialmente. Durante la presentación de una tarea (task) en clase le preguntaron al/ a la docente por el uso correcto de los verbos en infinitivo y en gerundio, y el/la docente procedió a explicarles y remitió al grupo al taller EGAP donde habían estudiado el tema. En el cuestionario, una estudiante comentó “Me gustaría algo más tradicional de la mano de lo didáctico, es decir, ver teoría de forma concreta desde lo básico". Es evidente que el estudiantado, además de asumir una posición crítica frente al programa también se permite elaborar propuestas que contribuyan al aprendizaje de la lengua extranjera.

\section{| Discusión}

Esta investigación se ha centrado en las voces del estudiantado de una universidad pública del área de la salud, en relación con la política lingüística de la institución. Sus voces han permitido dar cuenta no solo de los diferentes procesos de interpretación y apropiación que se han dado en la puesta en marcha del programa, sino también del lugar de árbitro que ejerce el estudiantado en la política lingüística.

Las diferentes respuestas del estudiantado evidencian su lectura y comprensión del programa, y su nivel de aceptación de la política. Estas se encuentran reflejadas en sus manifestaciones sobre el nivel de acuerdo, en relación con la elección del inglés como la lengua extranjera a estudiar en su formación en el pregrado, y la inclusión de los cinco niveles de inglés en los planes de estudio. Igualmente, se podría manifestar que la mayoría de los y las estudiantes se acomodan a la política lingüística al cumplir con las tareas, talleres, actividades y ejercicios que se plantean en el programa de inglés. Por lo anterior, se plantea que esta buena recepción y disposición frente la política lingüística institucional puede obedecer a las necesidades de formación en lengua extranjera particulares del área y a sus intereses personales. Es decir, el acceso a la literatura científica del área y las posibilidades de movilidad internacional demandan del estudiantado un buen nivel de lengua extranjera, particularmente del inglés. Además, se evidencia en sus palabras que valoran en gran medida la formación en inglés, por considerar que es un valor agregado a su formación profesional con implicaciones positivas para su vida académica y personal.

Aparte de lo anterior, teniendo en cuenta que la evaluación es uno de los componentes de la política lingüística en educación, en el que se establece la manera y lo que se ha de evaluar (Liddicoat, 2004), en esta investigación se presentan las voces del estudiantado dando cuenta de la manera como ellos y ellas han apropiado este componente. El documento maestro del programa institucional plantea el portafolio como uno de los procedimientos evaluativos porque responde a la importancia que se le da al carácter formativo de la evaluación. Para el estudiantado dicha estrategia evaluativa les ha permitido reforzar lo aprendido en clase, además de explorar otros temas de manera independiente. Al respecto, se plantea que, si bien algunos estudios en el contexto de la política lingüística se han centrado en las percepciones del estudiantado en el contexto universitario (Kagwesage, 2012), sobre el reconocimiento del aprendizaje de idiomas en su formación profesional (Pereira, 2013) y el aprendizaje del inglés y su compromiso (Jiménez 2018), en esta investigación se traen las voces de un estudiantado que da cuenta de las fortalezas de la política lingüística y, muy en particular, su aceptación y reconocimiento frente a la evaluación del programa, la cual propende por una evaluación formativa, distanciándose de alguna manera de la evaluación tradicional que, por lo general, ha caracterizado los ambientes universitarios. 
Es importante resaltar que, en el ámbito de la enseñanza y aprendizaje de las lenguas, el portafolio es una estrategia de evaluación alternativa ampliamente utilizada (Fox, 2017) debido a los beneficios en el aprendizaje y desarrollo de la autonomía del estudiantado (Pujolá, 2019). En este sentido, las voces del estudiantado del área de la salud han dado cuenta de lo valioso del portafolio en su proceso de aprendizaje de la lengua extranjera. En consecuencia, se podría señalar que este estudio permite conocer, desde la vivencia del estudiantado, su percepción sobre el componente de la evaluación en la política lingüística y en particular el uso del portafolio en dicha política, lo cual hasta el momento no se ha encontrado en otros estudios.

En contraste, este estudio ha encontrado que en el proceso de apropiación se evidencian diversas manifestaciones de resistencia frente a varios aspectos de la política lingüística. En la revisión de la literatura realizada hasta ahora, algunos estudios dan cuenta de los retos que enfrenta el estudiantado indígena en la puesta en marcha de la política lingüística (Usma et al., 2018) y en el aprendizaje de las lenguas extranjeras (Arismendi et al., 2016), pero poco se ha escuchado de la resistencia del estudiantado en la educación superior sobre la puesta en marcha de la política lingüística. Al respecto, esta investigación contribuye a la literatura existente al presentar las voces del estudiantado universitario, señalando los aspectos de la política con los cuales no conversan o hay desencuentros.

Respecto al componente de la evaluación se evidencia una tensión con el taller EGAP, el cual es uno de los instrumentos de evaluación en los cursos de inglés. Aunque el programa le apunta a una evaluación formativa, el contenido del taller y la manera como se ha presentado e implementado en el aula de clase ha generado en el estudiantado un sentimiento de contrariedad. Frente a la resistencia del estudiantado, Huang (2018) plantea en su estudio que los y las estudiantes se resisten al currículo y la pedagogía al encontrarlos poco útiles, sin embargo, no se evidencia resistencia del estudiantado en cuanto a la evaluación.

Kaplan \& Baldauf (2002) plantean la metodología como una de las áreas en la planeación de política en educación, por lo cual al inicio se presentó que el programa institucional se ha acogido a la enseñanza basada en tareas (TBLT). Con respecto a la metodología y asuntos curriculares, Gómez (2017) analiza las creencias sobre el aprendizaje del inglés de estudiantes en educación terciaria, en aras de mejorar la propuesta curricular. Aunque Gómez presenta las voces del estudiantado, dando cuenta de elementos relacionados con estrategias de aprendizaje, no hay evidencia de resistencia del estudiantado frente a aspectos curriculares o metodológicos, como sí lo evidencian las voces del estudiantado de este estudio, quienes han manifestado su desacuerdo con la metodología del/de la docente del curso. En consecuencia, este estudio plantea que esta posición crítica del estudiantado refleja que ellos y ellas no son simplemente agentes de la política lingüística, sino que, a su manera, el estudiantado asume el lugar de árbitro de la política por el grado de poder que ejerce en la implementación del programa. Igualmente, se observa el agenciamiento del estudiantado al proponer a sus docentes la realización de algunas actividades en clase, tales como el uso de canciones en inglés o la realización de juegos a través de aplicaciones como Kahoot.

Además de lo anterior, en relación con la inasistencia a clase y actitudes poco proactivas de algunos y algunas estudiantes en el aula de clase, se puede plantear que, aunque el estudiantado reconoce la importancia del inglés, hay quienes no logran asumir totalmente la responsabilidad y el compromiso necesario para su aprendizaje. Además, esta situación evidencia lo que plantea Johnson (2013a) en relación con la apropiación de la política lingüística, al señalar que en ocasiones se puede dar un rechazo implícito de la misma; es decir, en este caso el estudiantado aparentemente obedece o acepta el programa de formación en lengua extranjera, pero sus actitudes en clase reflejan lo contrario. 
Finalmente, esta investigación quisiera señalar dos puntos en particular. En primer lugar, este estudio aporta a la literatura sobre la política lingüística en educación, puesto que Liddicoat \& Baldauf (2008) han planteado la necesidad de estudios sobre los elementos del nivel micro de la política, es decir, en cuanto a la implementación de la misma y su relación con los diferentes agentes a nivel local. En este caso, se presenta la manera como un grupo de estudiantes del área de la salud se ha relacionado con la política establecida en la institución, donde se forma su interpretación y apropiación. Y, en segundo lugar, la presente investigación evidencia el agenciamiento del estudiantado en la puesta en marcha de la política lingüística. Así pues, se enfatiza que el estudiantado no es un simple implementador de la política lingüística, sino que también ejerce algún grado de poder en la puesta en marcha de la política en el aula de clase; es decir, el estudiantado es también, en mayor o menor medida, árbitro de la política lingüística, lo cual poco se ha analizado en otros estudios sobre política lingüística en educación. Por tanto, el estudiantado no es solamente un agente que sigue las instrucciones de sus profesores/as en el aula de clase, sino que también propone iniciativas y asume posiciones críticas que le dan su lugar de árbitro, tal como lo expresan Johnson \& Johnson (2014). Los autores plantean que el árbitro de la política lingüística es cualquier actor de la política -profesores, administradores, creadores de la política, entre otros-, que ejerza algún grado de poder sobre la manera como la política es creada, interpretada o apropiada en un contexto determinado.

Por consiguiente, los hallazgos presentados en esta investigación permiten considerar algunas implicaciones. Teniendo en cuenta que la universidad le ha apostado a la formación en lengua extranjera de todo el estudiantado de la institución, se hace preciso que la administración del programa busque estrategias que le permitan conocer de manera amplia cómo el estudiantado de la institución se ha relacionado con el programa institucional, lo cual informará sobre ese proceso de apropiación del estudiantado. Igualmente, es importante considerar una revisión de esos elementos de la propuesta evaluativa del programa que han generado ruido en la población estudiantil, de manera que se fortalezca la evaluación formativa y alternativa que plantea el documento maestro. Por último, es preciso promover las investigaciones de política lingüistica en educación que den lugar a las voces del estudiantado universitario, las cuales han sido poco escuchadas cuando se trata de su propia formación, y muy en particular sobre las decisiones sobre la inclusión de una lengua extranjera como el inglés en su proceso de formación profesional.

\section{| Conclusiones}

Teniendo en cuenta las voces del estudiantado del área de la salud de una universidad pública colombiana, sobre la implementación de la política en lengua extranjera, se exponen las siguientes conclusiones.

En primer lugar, se halló, por medio de los cuestionarios y la observación de clase, que el estudiantado valora la formación en una lengua extranjera como el inglés, ya que le representa un beneficio para su vida personal, académica y laboral; al tiempo que manifiesta su apoyo a la inclusión del inglés en los planes de estudio de los pregrados de la institución. Sumado a eso, el estudiantado concibe el programa institucional como la oportunidad de formarse en inglés, reconoce que su experiencia en las clases ha sido positiva, y considera, además, que la metodología es innovadora. 
Esta actitud positiva hacia la lengua extranjera en el plan de estudios y, en particular, hacia el programa, corrobora que existe una tendencia por parte del estudiantado a percibir el inglés como un saber con un alto valor para su desarrollo personal. Por otro lado, los comentarios hacia el programa institucional revelan que se considera innovador, probablemente por su enfoque centrado en el/la estudiante y en la practicidad de las tareas y la comunicación, que son características del aprendizaje basado en tareas, centro del plan curricular. Así mismo, es posible concluir que a la buena aceptación de las tareas se le suma una serie de aspectos que también influyen en el éxito de la aplicación de una metodología en concreto. Entre dichos aspectos, se encuentran las interacciones y los roles de los agentes en el aula, los estilos de enseñanza y aprendizaje que converjan en ella, el nivel de proficiencia del/de la docente, entre otros. Es probable que esta respuesta de los y las estudiantes frente a la enseñanza basada en tareas sea una forma de reaccionar frente a una metodología que se adecúa más a sus necesidades educativas y de mayor consonancia identitaria con las nuevas generaciones.

En segundo lugar, se observa que la mayoría de los y las estudiantes realizan las actividades propuestas en clase y valoran el portafolio, porque reconocen que este promueve su autonomía en el aprendizaje. Estas actitudes se traducen como un alto grado de aceptación en los procesos de apropiación de la política lingüística. No obstante, también hay respuestas del estudiantado que demuestran cierta resistencia. Un ejemplo de ello es la reacción que presentan frente al taller EGAP, uno de los instrumentos de evaluación del programa. Ellos y ellas manifiestan que este tiene poca coherencia con la metodología del curso y sus contenidos. Adicionalmente, se evidencia la resistencia de algunos y algunas estudiantes con su actitud poco proactiva y participativa en el aula de clase, además de la impuntualidad y poca asistencia a clase, lo cual fue recurrente especialmente en la Facultad de Veterinaria. Igualmente, se encontró resistencia frente a la metodología del o de la docente, por ejemplo, respecto al manejo de la corrección del error. El estudiantado indicó que esperaba correcciones inmediatas para mejorar en el aprendizaje, creencia con la que un docente no estaba de acuerdo. También, el estudiantado manifiesta insatisfacción ante el uso constante del material propuesto en el programa, puesto que parece que algunos y algunas docentes hacen de este material su única guía en la práctica pedagógica en el aula.

Lo anterior, conduce a pensar que hay un cierto grado de inconsistencia en las voces del estudiantado. Por un lado, valoran la política en lengua extranjera, porque la consideran de gran beneficio para su vida y realizan las actividades; pero, por otro lado, no todos y todas asumen con responsabilidad y compromiso esta oportunidad de formación.

Existen varios motivos que podrían explicar el comportamiento del estudiantado de aceptación y resistencia paralelas. Por un lado, la resistencia aparece frente a una evaluación: el taller EGAP. La inconformidad del estudiantado puede señalar que la evaluación tradicional, estructuralista y descontextualizada -características atribuidas a este taller- ya no es tolerable para una generación hiper-estimulada como la actual, más aún cuando este va en discordancia con el resto de la metodología del curso. En este mismo sentido, se podría plantear que hay un desencuentro entre las creencias del estudiantado, en cuanto a la corrección del error y las creencias de algunos o algunas docentes.

De otro lado, el comportamiento desinteresado de algunos/algunas estudiantes podría deberse a diversas razones. Su inasistencia a clase y el aparecer únicamente para los exámenes podría entenderse como una señal de que no están interesados en participar de la clase, porque consideran que tienen un mejor nivel del que se enseña o porque consideran que el inglés no es importante, tal vez porque perciben la lengua extranjera como un "relleno" en el plan de estudios y no algo por lo que deberían ocuparse en su labor académica. 
La motivación estudiantil también es un factor que hay que tener en cuenta al momento de evaluar el porqué del desinterés en los contenidos del curso. Es posible que los docentes no estén generando prácticas que eleven el nivel de motivación en los alumnos, por lo que se hace preciso recordar que el rol del profesorado es esencial para mantener e incrementar la motivación del estudiantado en el aprendizaje de una lengua extranjera. Tal como se encuentra planteado en el documento maestro de la política lingüística, el profesorado del programa debe motivar al estudiantado para que asuma la responsabilidad de invertir buen tiempo al desarrollo de las tareas, además debe ser un apoyo en el proceso de aprendizaje.

Asimismo, cabe resaltar la necesidad de que el profesorado del programa busque un equilibrio entre la motivación intrínseca y la motivación extrínseca del estudiantado. Preguntar por los temas que le gustan al estudiantado, identificar sus intereses para abordarlos en clase, crear un buen ambiente para el aprendizaje y para el proceso de evaluación pueden ser ejemplos de la motivación intrínseca. Además, explorar diversas estrategias didácticas, tales como realizar dinámicas que promuevan la interacción entre el estudiantado, trabajar con música y dar retroalimentación positiva, puede también aportar a la motivación intrínseca. Por último, es preciso que el profesorado genere espacios de reflexión con el estudiantado sobre su formación y el lugar de las lenguas extranjeras en este proceso, de manera que este tome conciencia y saque el mejor provecho de la política lingüística institucional.

\section{| Referencias}

Alagozlu, N. (2012). English as a foreign language cul-de-sac in Turkey. Procedia- Social and Behavioral Sciences, 47. https://doi.org/10.1016/j.sbspro.2012.06.896

Alonso, J., Martin, J., y Gallo, B. (2015). El nivel de inglés después de cursar la educación superior en Colombia: una comparación de distribuciones. Revista de Economía Institucional, 17(33), 275-298. https://doi.org/10.18601/01245996.v17n33.12

Angrosino, M.V. (2015). Recontextualización de la observación: Etnografía, pedagogía y las perspectivas de una agenda política progresista. En N. K. Denzin \& Y. S. Lincoln (Eds.), Manual de investigación cualitativa V.IV: Métodos de recolección y análisis de datos (pp. 203-234). Gedisa Editorial.

Arismendi F., Ramírez D., y Arias S. (2016). Representaciones sobre las lenguas de un grupo de estudiantes indígenas en un programa de formación de docentes de idiomas. Colombian Applied Linguistics Journal, 18(1), 84-97.

Bretxa, V., \& Vila, X. (2015). Language policy in higher education: The case of medium size languages. Multilingual Matters.

Bermúdez, J., Fandiño, Y., y Ramírez, A. (2014). Percepciones de directivos y docentes de instituciones educativas distritales sobre la implementación del Programa Bogotá Bilingüe. Voces y Silencios: Revista Latinoamericana de Educación, 5(2), 135-171.

Canagarajah, S. (2006). Ethnographic methods in language policy. En T. Ricento (Ed.), An introduction to language policy: Theory and method (pp. 153-169). Blackwell.

Canale, G. (2015). Mapping conceptual change: The ideological struggle for the meaning of EFL in Uruguayan education. L2 Journal, 7(3), 15-39. http://doi.org/10.5070/L27323707

Denzin, N., \& Lincoln, Y. (2008). Strategies of qualitative inquiry. Sage Publications.

Denzin, N., \& Lincoln, Y. (2018). The sage handbook of qualitative research (5a ed.). SAGE Publications.

Díaz, C., Alarcón, P., \& Ortiz, M. (2015). A case study on EFL teachers' beliefs about the teaching and learning of English in public education. Porta Linguarum, 23, 171-186. http://www.ugr.es/ portalin/articulos/PL_numero23/11\%20\%20Claudio\%20Diaz.pdf

Dornyei, Z., \& Taguchi, T. (2010). Questionnaires in second language research:Construction, administration, and processing. Routledge. 
Fallon, G., \& Rublick, N. (2012). Second-language education policy in Quebec: ESL teachers' perceptions of the effects of the policy of English as a compulsory subject at the early primary level. TESOL Canada Journal, 29(2), 58-73. https://teslcanadajournal.ca/index.php/tesl/article/view/1100/919

Fishman, J. (2002). Endangered minority languages: Prospects for sociolinguistic research. International Journal on Multicultural Societies, 4(2), 270-275. www.unesco.org/shs/ijms/vol4/issue2/art7

Fox, J. (2017). Using portfolios for assessment/alternative assessment. En E. Shohamy, I. Or \& S. May (Eds.), Language testing and assessment (pp.135-147). Springer.

Fuentes, R. (2019). English learners' appropriation of English language policy at a U.S. university. International Multilingual Research Journal, 14(3), 233-247. https://doi.or$\mathrm{g} / 10.1080 / 19313152.2019 .1684422$

Gómez, J. F. (2017). Creencias sobre el aprendizaje de una lengua extranjera en el contexto universitario. Íkala, Revista de Lenguaje y Cultura, 22(2), 203-219.

Jiménez, P. K. (2018). Exploring students' perceptions about English learning in a public university. HOW, 25(1), 69-91. https://doi.org/10.19183/how.25.1.385

Heck, R. (2004). Studying educational and social policy, theoretical concepts and research methods. Lawrence Erlbaum Associates.

Hornberger, N., \& Johnson, D. (2007). Slicing the onion ethnographically: Layers and spaces in multilingual language education policy and practice. TESOL Quarterly, 41(3), 509-532. https://doi.org/10.1002/j.1545-7249.2007.tb00083.x

Hornberger, N., \& Johnson, D. (2011). The ethnography of language policy. En T. L. McCarty (Ed.), Ethnography and language policy (pp. 273-289). Routledge.

Huang, Y. (2018). Learner resistance to English-medium instruction practices: A qualitative case study. Teaching in Higher Education, 23(4), 435-449. https://doi.or-

g/10.1080/13562517.2017.1421629

Johnson, B., \& Christensen, L. (2008). Educational research: Quantitative, qualitative, and mixed approaches. SAGE Publications, Inc.

Johnson, D. (2013a). Language policy. Palgrave Macmillan.

Johnson, D. (2013b). Positioning the language policy arbiter. En J.W. Tollefson (Ed.), Language policies in education, critical issues (pp. 116-236). Routledge.

Johnson, D., \& Johnson, E. (2014). Power and agency in language policy appropriation. Language Policy, 13(3). https://education.wsu.edu/documents/2015/03/312.pdf

Jung, S., \& Norton, B. (2002). Language planning in Korea: The new elementary English program. En J. W. Tollefson (Ed.), Language policies in education: Critical issues (pp. 245-265). Lawrence Erlbaum Associates.

Kachru, B. B., \& Nelson, C. L. (2001). World Englishes. En A. Burns \& C. Coffin (Eds.), Analysing English in a global context (pp. 9-25). Routledge.

Kagwesage, A.M. (2012). Higher education students' reflections on learning in times of academic language shift. International Journal for the Scholarship of Teaching and Learning, 6(2). https://doi.org/10.20429/ijsotl.2012.060218

Kaplan, R., \& Baldauf, R. (2002). Language and language-in-education planning in the Pacific Basin. Springer.

Liddicoat, A. (2013). Language-in-education policies. Multilingual Matters.

Liddicoat, A. J. (2004). Language policy and methodology. International Journal of English Studies, 4(1), 153-171. https://revistas.um.es/ijes/article/view/48221

Liddicoat, A., \& Baldauf, R. (2008). Language planning and policy: Language planning in the local contexts. Multilingual Matters.

McCarty, T. (2011). Ethnography and language policy. Routledge.

McCarty, T. (2015). Ethnography in language planning and policy research. En F.M. Hult \& D.C. Johnson (Eds.), Research methods in language policy and planning (pp. 81-106). Wiley Blackwell. 
Menken, K., \& García, O. (2010). Negotiating language policies in schools: Educators as policymakers. Routledge.

Merriam, S. (1998). Qualitative research and case study applications in education. John Wiley \& Sons.

Ministerio de Educación Nacional -MEN-. (2005). Programa Nacional de Bilingüismo. Colombia 2004-2019. http://www.colombiaaprende.edu.co/html/productos/1685/article-158720.html

Miranda, N. (2018). Política educativa para el bilingüismo español-inglés en Colombia: Rastreo de su trayectoria desde las aulas (Tesis doctoral). Universidad del Quindío.

Montoya, A. (2013). La incidencia de las políticas y la planeación lingüísticas en las actitudes lingüisticas de los estudiantes colombianos. Forma y Función, 26(1), 237-260. http://www.scielo.org.co/scielo.php?script=sci_arttext\&pid=S0120338X2013000100010\&lng=en\&tlng=es

Muñoz, M. (2019). Las actitudes y creencias lingüísticas de estudiantes universitarios. Revista Iberoamericana para la Investigación y el Desarrollo Educativo, 9(18), 748-767. https://doi.org/10.23913/ride.v9i18.444

Peláez, O., \& Usma, J. (2017). The crucial role of educational stakeholders in the appropriation of foreign language education policies: A case study. Profile Issues in Teachers' Professional Development, 19(2), 121-134. https://doi.org/10.15446/profile.v19n2.57215

Pereira, S. (2013). Planificación y políticas lingüísticas en la enseñanza de extranjeras a nivel universitario: Un análisis de percepciones. Lenguaje, 41(2), 383-406. https://doi.org/10.25100/lenguaje.v41i2.4975

Pujolá, J. T. (2019). El portafolio digital en la docencia universitaria. Ediciones Octaedro, S.L.

Roldán, A. M., y Peláez, Ó. (2017). Pertinencia de las políticas de enseñanza del inglés en una zona rural de Colombia: Un estudio de caso en Antioquia. Íkala. Revista de Lenguaje y Cultura, 22(1), 121-139. https://doi.org/10.17533/udea.ikala.v22n01a08

Seidlhofer, B. (2011). Understanding English as an international language. Oxford University Press.

Shohamy, E. (2006). Language policy: Hidden agendas and new approaches. Routledge.

Tejada, H., y Samacá, G. (2012). Algunos efectos del discurso dominante en las actitudes y expectativas de directivos docentes frente al programa nacional de bilingüismo (PNB). Lenguaje, 40(2), 415-445. https://doi.org/10.25100/lenguaje.v40i2.4956

Tseligka, T. (2016). Developing a foreign language policy in Greek higher education (HE): Striving between Scylla and Charybdis. International Journal of Language, Translation and Intercultural Communication, 4(1), 54-67. https://doi.org/10.12681/ijltic.10352

Tsuchiya, K., \& Pérez, M. (2015). Comparing the language policies and the students' perceptions of CLIL in tertiary education in Spain and Japan. Latin American Journal of Content and Language Integrated Learning, 8(1), 25-35. https://doi.org/10.5294/laclil.2014.8.1.3

Villalobos, G. M., Arciniegas, A. M., \& González, C. A. L. (2016). Formación docente en TIC con el Centro de Innovación Educativa CIER-SUR. Trilogía Ciencia Tecnología Sociedad, 8(14), 65-80.

Usma, J., Ortíz, J., \& Gutiérrez, C. (2018). Indigenous students learning English in higher education: Challenges and hopes. Ikala Revista de Lenguaje y Cultura, 23(2). https://aprendeenlinea.udea.edu.co/revistas/index.php/ikala/article/view/330989

Wang, H. (2008). Language Policy Implementation: A look at teachers' perceptions. Asian EFL Journal, 29, 1-25. http://www.asian-efl-journal.com/pta_August_08.pdf

Widdowson, H.G. (1997). The forum: EIL, ESL and EFL: Global issues and local interests. World Englishes, 16(1), 135-146. https://doi.org/10.1111/1467-971X.00054 
Apéndice A

\section{Árbitros de las políticas lingüísticas: docentes y estudiantes en los procesos de interpretación y apropiación del programa de formación en lengua extranjera}

Apreciado y apreciada estudiante le solicitamos muy cordialmente responder el siguiente cuestionario, el cual hace parte de la investigación "Árbitros de las políticas lingüísticas: docentes y estudiantes en los procesos de interpretación y apropiación del programa de formación en lengua extranjera de la Universidad", cuyo objetivo principal es analizar los procesos de interpretación y apropiación de la política lingüística, por parte de los/las docentes de inglés y estudiantes de pregrado de la universidad.

La información suministrada será utilizada sólo para propósitos investigativos, ésta y su nombre serán mantenidos bajo estricta confidencialidad, por lo tanto, su privacidad será respetada.

El cuestionario consta de dos partes: la primera sección es acerca de su experiencia en el programa de inglés en la Universidad y la segunda abarca información demográfica. Favor responder cada una de las preguntas de la manera más objetiva y sincera posible, todas sus respuestas son importantes para este proyecto, por tanto, no hay respuestas correctas o incorrectas. Les agradecemos de antemano su valiosa colaboración.

I. El programa institucional en formación extranjera en inglés

A continuación, queremos conocer sus apreciaciones en relación con el programa institucional y su experiencia en los cursos de inglés de la Universidad.

1. ¿Qué viene a su mente cuando escucha programa de formación en inglés?

2. ¿Cómo describiria su experiencia en el curso de inglés o en los cursos de inglés que ha tomado

en

el

programa?

3. ¿Cómo describiría usted la metodología del curso de inglés del programa?

4. Señale los métodos evaluativos que se han aplicado en las clases de inglés del programa. (Puede seleccionar todos los que aplican):

a) Quiz

b) examen parcial

c) examen final

d) talleres

e) portafolio

f) presentación oral individual

g) Proyecto 

h) tarea (task)
i) exposición
j) Entrevista
k) Otro ¿Cuál?

5. Señale los materiales que se usa en la clase de inglés. Seleccione todos los que aplican:

a) Video

b) Audio

c) Material del programa

d) fotocopias

e) libros de literatura

f) libros de inglés

g) Internet

h) Otro, ¿cuál?

A continuación, encontrará algunos de los principios que sustentan el programa de formación en lengua extranjera (inglés) de la Universidad. Por favor, seleccione el número que mejor representa su opinión según la siguiente escala:

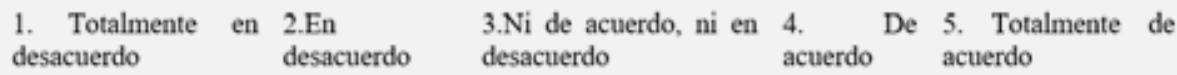

6. "El inglés se ha convertido en el lenguaje más usado en el ámbito académico y cientifico del mundo contemporáneo y por ello se ha elegido como la base de la Politica de lengua extranjera para estudiantes de pregrado de la Universidad"

$$
\begin{array}{llllll}
1 & 2 & 3 & 4 & 5
\end{array}
$$

7. "La Universidad decide incluir cinco niveles de inglés en los planes de estudio de los pregrados".

$$
\begin{array}{lllll}
1 & 2 & 3 & 4 & 5
\end{array}
$$

8. "El fin de la Política de lengua extranjera es mejorar el nivel de desempeño de los estudiantes, facilitar la interacción con el mundo académico y cientifico, fortalecer el acceso al mercado laboral y lograr una mayor competitividad de los egresados de la Universidad".

$$
\begin{array}{llllll}
1 & 2 & 3 & 4 & 5
\end{array}
$$

9. "La Universidad propone articular el inglés de manera transversal en cursos disciplinares en los programas de pregrado" (ofrecer cursos disciplinares en inglés).

$$
\begin{array}{lllll}
1 & 2 & 3 & 4 & 5
\end{array}
$$

10. "En el programa de inglés se espera que el estudiante esté en capacidad de hacer uso de la lengua de forma cada vez más independiente y cumpla con las tareas asignadas de forma autónoma".

$$
\begin{array}{lllll}
1 & 2 & 3 & 4 & 5
\end{array}
$$

11. En el programa el estudiante adquiere el control sobre su propio aprendizaje, planificando, tomando decisiones, buscando información y materiales, evaluando y rectificando su desempeño".

$$
\begin{array}{lllll}
1 & 2 & 3 & 4 & 5
\end{array}
$$

12. "La evaluación se considera una construcción social en la que participan el profesor y los estudiantes de manera dialogada"

$\begin{array}{lllll}1 & 2 & 3 & 4 & 5\end{array}$




\section{INTERPRETACIÓN Y APROPIACIÓN}

En el siguiente apartado, le solicitamos completar los enunciados de acuerdo a su opinión y experiencia en los cursos de inglés del programa de la Universidad:

13. E1 aprendizaje de una lengua extranjera como el inglés significa...

14. Como estudiante, $\mathrm{mi}$ rol en la clase de inglés...

15. Los materiales que yo uso en el aprendizaje del inglés son:

16. Las tareas (tasks) asignadas en el curso de inglés me parecen...

17. E1 portafolio lo hago de la siguiente manera:

18. En el curso de inglés considero que el taller EGAP...

19. Aparte de la clase, las actividades extracurriculares que hago son...

20. Algo que hace parte del curso de inglés y que yo no hago es...

21. Algo que me gusta del programa de inglés es...

22. Algo que NO me gusta del programa de inglés es...

\section{Caracterización demográfica de los y las estudiantes}

Seleccione la opción o las opciones que correspondan a su información personal:

23. Marque su rango de edad:
a) 15- 19 años
b) 20- 25 años
c) 26-30 años
d) 31-35 años
e) $36-40$ años
f) Más de 41 años

24. Indique el sexo:
a) Mujer
b) Hombre
c) Otro, ¿cuál?: 
25. ¿Pertenece a alguna etnia, grupo indígena o comunidad

afrocolombiana?
a) $\mathrm{Si}$ ¿a cuál?
b) No

26. Indique su lugar de procedencia (municipio y departamento).

27. Indique su estrato socioeconómico:
a. 1-2
b. $3-4$
c. $5-6$

28. Seleccione el tipo de institución de la cual egresó en el bachillerato:
a) Colegio público
b) Colegio privado
c) Otro ¿cuál?

29. ¿Hace cuánto egresó del bachillerato?
a. Menos de 1 año
b. Entre 1 y 3 años
c. Entre 4 y 6 años
d. Más de 7 años

30. Aparte del curso o de los cursos de inglés del programa de la Universidad, indique las oportunidades de formación en inglés que ha tenido anteriormente. Señale todas las opciones que aplican a su experiencia de formación.
a) Inglés en el bachillerato
b) Cursos de inglés en un centro de idiomas
c) Programa de inglés (en cd, en linea, libros, etc.)
d) Autoformación
e) Otro, ¿cuál?
f) No aplica 
31. Seleccione el semestre que cursa actualmente en la carrera:
a. Semestre 1
b. Semestre 2
c. Semestre 3
d. Semestre 4
e. Semestre 5
f. Otro, ¿cuál?

32. Teniendo en cuenta que el foco del estudio son sus experiencias en los procesos de interpretación y apropiación del programa de formación en lengua extranjera, si hay algo más que quisiera agregar, le pedimos escribirlo en el siguiente espacio:

\section{¡Muchas gracias!}

